\title{
Mudança no status sociométrico negativo de alu- nos com dificuldades de aprendizagem
}

\author{
Status sociométrico de alunos com dificuldades
}

\author{
Renata Cristina Moreno Molina \\ Almir Del Prette
}

\section{Resumo}

\begin{abstract}
Estudos que investigam a aceitação de alunos com dificuldades de aprendizagem mostram que estes apresentam status sociométrico negativo, indicando um repertório inadequado de habilidades sociais. $O$ presente trabalho buscou avaliar a promoção de habilidades sociais sobre o status sociométrico desses estudantes. Os participantes foram divididos em um grupo de intervenção $\mathrm{Gl}$ ( 6 alunos com dificuldades de aprendizagem) e dois grupos sem intervenção GCl e GC2 (6 e 12 alunos com e sem dificuldades de aprendizagem, respectivamente). Os alunos foram avaliados antes e depois da intervenção com o Protocolo de Indicação Sociométrica. O Treinamento em Habilidades Sociais com o Gl abrangeu 10 sessões de uma hora e meia cada. A comparação entre as avaliações revelou melhora no status de cinco participantes do $\mathrm{Gl}$ e em apenas dois participantes do $\mathrm{GCl}$. Os dados obtidos ressaltam a importância das habilidades sociais na produção de um status sociométrico positivo.
\end{abstract}

Palavras-chave: dificuldades de aprendizagem; avaliação psicológica; habilidades sociais.

\section{Change in negative sociometric status in students with learning disabilities}

\begin{abstract}
Studies that investigate the acceptance of learning disabilities student point out that they present negative sociometric status, indicating an inappropriate social skills repertorie. This work has evaluated the improvement of social skills in social status of these students. The participants were divided in to one intervention group Gl (6 learning disabilities students) and two without intervention group GCI e GC2 (6 and I2 students with or no learning disabilities, respectively). The students were evaluated before and after the intervention with Sociometric Indication Protocol. The Social Skills Training was included 10 sessions of one hour and a half. The comparison between the evaluations showed improvement in social status of five Gl participants and two of GCI. The results obtained show the importance of social skills in the production of a positive sociometric status.

Keywords: learning disabilities; psychological assessment; social skills.
\end{abstract}

\section{Cambio en el status sociométrico negativo de alumnos con dificultades de aprendizaje}

\section{Resumen}

Estudios que investigan la aceptación de alumnos con dificultades de aprendizaje muestran que ellos presentan un status sociométrico negativo, indicando un repertorio inadecuado de habilidades sociales. Este trabajo buscó evaluar la promoción de habilidades sociales sobre el status sociométrico de esos estudiantes. Los participantes fueron divididos en un grupo sin intervención Gl (6 alumnos con dificultades de aprendizaje) y dos grupos sin intervención GCl y GC2 (6 y 12 alumnos con y sin dificultades de aprendizaje respectivamente). Los alumnos fueron evaluados antes y después de la intervención con el Protocolo de Indicação Sociométrica. El entrenamiento en Habilidades Sociales con el Gl abarcó 10 sesiones de una hora y media cada una. La comparación entre las evaluaciones reveló una mejoría en el status de cinco participantes del Gl y en apenas dos participantes del GCl. Los datos obtenidos resaltan la importancia de las habilidades sociales en la producción de un status sociométrico positivo.

Palabras clave: dificultades de aprendizaje; evaluación psicologica; habilidades sociales. 


\section{Introdução}

Nas últimas décadas, as explicações referentes à ocorrência do fracasso escolar em nosso meio foram relacionadas a diferentes terminologias utilizadas no âmbito educacional, sendo distúrbios e dificuldades de aprendizagem as mais comuns. No termo distúrbio de aprendizagem se encontra a idéia de se associar o fracasso escolar a características do aluno e de sua família. Por outro lado, sob o termo dificuldade de aprendizagem, a ênfase do fracasso escolar está relacionada à discrepância entre o que se presume que uma criança seja capaz de aprender potencialmente, sob uma dada situação normal de sala de aula, e o que ela efetivamente realiza (Almeida e colaboradores, 1995; Weiss, 1992).

Sánchez (2004) e Sisto, Boruchovitch, Fini, Brenelli e Martinelli (2004), em uma revisão sobre o tema, diferenciam os alunos que apresentam episódios de fracasso escolar, caracterizados como um baixo rendimento, devido a causas extrínsecas (como ensino inadequado, baixa motivação e fatores econômicos), dos que revelam ter dificuldades de aprendizagem originadas por fatores intrínsecos (caracterizadas por discrepâncias no desenvolvimento dos processos psicológicos como a percepção, a atenção e a memória).

Independentemente de quais aspectos estão envolvidos para dificultar o processo de aprendizagem, deve-se considerar que a superação do fracasso escolar demanda esforços conjuntos de profissionais visando não apenas a melhoria dos métodos pedagógicos de ensino, mas também a alteração de outros fatores do contexto de sala de aula, como a qualidade das relações sociais entre os alunos e destes com o professor.

As interações sociais de qualidade são entendidas como educativas na medida em que representam condições para a aquisição de conceitos, habilidades e estratégias cognitivas que afetam o desenvolvimento social e a aprendizagem (Davis, Silva \& Espósito, 1989; Gresham, 2001; Pontecorvo, sd; Romero, 1995; Vigotsky, 2003). Para Del Prette e Del Prette (200I) e Vigotsky (2003), a aprendizagem é um processo de construção do conhecimento que ocorre na interação do sujeito com a família, com a escola e com a sociedade. Assim, dificuldades neste processo, que levam ao fracasso escolar, podem ser consideradas como decorrentes "de uma possível constelação de fatores de ordem pessoal, familiar, emocional, pedagógica e social que só adquirem sentido quando referidos à história de relações e interações do sujeito com seu meio, inclusive e, sobretudo, o escolar" (Almeida e cols., 1995, p.122).

A compreensão das interações estabelecidas pelos alunos e de seus produtos acadêmicos requer uma análise das características (cognitivas, afetivas e comportamentais) que influenciam esse processo (Gresham, 200I; Saint-Onge, 1999). Uma abordagem voltada para a promoção do desenvolvimento interpessoal dos indivíduos é a das Habilidades Sociais, entendidas por vários autores (Del Prette \& Del Prette, 200I; 2005; Gresham, 200I) como diferentes classes de comportamentos sociais do repertório do indivíduo, que contribuem para a competência social, favorecendo um relacionamento produtivo e saudável com as pessoas. A competência social é definida, por esses autores, como a capacidade de articular pensamentos, sentimentos e ações em função de objetivos pessoais e das demandas da situação e da cultura, gerando conseqüências positivas para o indivíduo e para a sua relação com as demais pessoas. Os critérios de funcionalidade, que definem um comportamento como socialmente competente ou não, supõem a capacidade do indivíduo em articular as habilidades sociais em um desempenho social que atende às demandas da situação e da cultura.

$\mathrm{Na}$ infância, um repertório elaborado de habilidades sociais contribui decisivamente para a manutenção de relações harmoniosas com colegas e adultos sendo o status social, o julgamento positivo por outros significantes, além de alguns comportamentos adaptativos, como rendimento acadêmico, importantes para a avaliação da competência social (Del Prette \& Del Prette, 2005; Merrell, 1993; Newcomb, Bukowski, \& Patee, 1993). Para Caldarella e Merrell (1997), Gresham (200I) e Gresham e Elliott (1990), as crianças que apresentam comportamentos sociais apropriados reúnem um conjunto de habilidades sociais importantes para o sucesso das interações sociais em idade escolar: oferecer ajuda, cumprimentar, 
juntar-se a grupo de amigos em brincadeira, demonstrar empatia, demonstrar senso de humor, controlar as emoções, seguir regras, cooperar com outros, aceitar críticas, iniciar conversação etc.

Conforme alguns autores (Del Prette \& Del Prette, 2005; Gresham, 1995; Malecki \& Elliott, 2002; McClelland, Morrison \& Holmes, 2000), as crianças que apresentam dificuldades em habilidades sociais, definidas como déficits de aquisição ou de desempenho adequados às demandas da situação e da cultura, comprometem sua competência social de duas formas: na forma encoberta de incômodo, mágoa, ressentimento, ansiedade acompanhada de esquiva ou fuga das demandas interpessoais (reações não habilidosas passivas ou reações passivas); na forma aberta de agressividade física ou verbal, negativismo, ironia, autoritarismo ou coerção (reações não habilidosas ativas ou reações agressivas).

O repertório de habilidades sociais de crianças pode ser visto também como um fator para o sucesso acadêmico, dada a constatada associação entre características interpessoais negativas e dificuldades ou distúrbios de aprendizagem (Gresham, 1992; Gresham \& Elliott, 1989; Vaughn \& Hogan, 1990; Swanson \& Malone, 1992). Pesquisas nesta área (Del Prette \& Del Prette, 2003; Molina \& Del Prette, 2002; O'Shaughnessy, Lane, Gresham \& BeebeFrankenberger, 2002; Romero, 1995) têm mostrado que as crianças com distúrbios ou dificuldades de aprendizagem apresentam características interpessoais que incluem, entre outros aspectos, tendência à agressividade, a interações negativas com companheiros, a apresentarem mais problemas de personalidade, menos comportamentos orientados para tarefa e um repertório menos elaborado de comportamentos interpessoais apropriados e desejáveis socialmente. Em tarefas escolares, Romero (1995) afirma que os alunos com dificuldades de aprendizagem se distraem mais (apresentando índices baixos de atenção sustentada) e são mais dispersivos (levantam-se, falam inoportunamente etc.) que os seus colegas sem dificuldades de aprendizagem, interagem mais com seus professores, exigindo maior atenção (embora esta tenha caráter corretivo, em respostas às distrações) e participam menos das iniciativas de grupo.
Essa caracterização negativa do desempenho social de crianças com dificuldades de aprendizagem aparece na avaliação de seus pais (Marturano, Linhares \& Parreira, 1993; Melo, 2004), professores (Fad, 1989; Maluf \& Bardelli 1991; Marturano \& Loureiro, 2003) e colegas (Stone \& La Greca, 1990; Vaughn \& Hogan, 1990; Vaughn, Mclntosh \& Spencer-Rowe, 199I), sendo a destes últimos a que mais tem recebido atenção da literatura, já que os pares fornecem uma importante perspectiva do repertório comportamental de crianças em idade escolar.

Alguns autores (Garcìa \& Musitu, 1993; Parker \& Asher, 1987; Stone \& La Greca, 1990) têm mostrado que as informações que o estudante recebe de seus pares repercute em sua auto-avaliação social e afeta o desempenho acadêmico, além de predizer problemas posteriores como ajustamento, sucesso escolar e no trabalho. Estudos que investigam a aceitação por pares na escola (Asher \& Coie, 1990; Gresham, Sugai \& Horner, 200I; Parker \& Asher, 1987; Stone \& La Greca, 1990; Vaughn \& Hogan, 1990; Vaughn, Mclntosh \& Spencer-Rowe, 199I) têm mostrado que os alunos com dificuldades de aprendizagem são menos aceitos que seus colegas com bom desempenho acadêmico.

Um procedimento muito utilizado em pesquisas para medir o status social, a popularidade e aceitação/ rejeição de alunos por seus colegas de sala é a avaliação sociométrica. Alguns procedimentos sociométricos permitem a identificação das crianças rejeitadas, negligenciadas, populares e controvertidas da escola e destacam as seguintes características interpessoais associadas a esse status (Coie, Dodge, \& Coppotelli, 1982; Frentz, Gresham, \& Elliott, 1990):

- Populares: alunos vistos como cooperativos e líderes e que tendem a exibir mais comportamentos sociais apropriados;

- Rejeitados: alunos mais disruptivos e briguentos, menos cooperativos e com poucas características de liderança;

- Controvertidos: alunos que tendem a oscilar entre comportamentos sociais apropriados e comportamentos disruptivos;

- Negligenciados: alunos que apresentam timidez, isolamento social e são ignorados em sala. 
Coie, Dodge e Coppotelli (1982) avaliaram a aceitação de crianças em idade escolar por meio do procedimento de Indicação Sociométrica, o qual consiste no registro dos nomes dos colegas mais queridos e dos menos queridos em um determinado grupo, permitindo a sua classificação em um dos status sociométricos apresentados anteriormente. Os estudos que utilizaram este sistema de classificação encontraram os estudantes com dificuldades de aprendizagem bastante representados no status rejeitado e negligenciado e pouco representados no status popular (Asher \& Coie, 1990; Bryan \& Sherman, 1980; Stone \& La Greca, 1990; Vaughn \& Hogan, 1990; Vaughn, Mclntosh \& Spencer-Rowe, 1991).

Para Asher e Rose (1999), uma explicação plausível para o status negativo é que as dificuldades de aprendizagem tornam-se visíveis aos colegas da sala nos primeiros dias do semestre letivo e, como as habilidades acadêmicas são bastante valorizadas pelo professor, a rejeição dos alunos estaria condicionada a um baixo desempenho em tarefas escolares. Por outro lado, para Coie e Dodge (1983), Del Prette e Del Prette (2005) e Gresham, Sugai e Horner (200I), a representação em um destes status negativos seria um indicativo de um repertório inapropriado de habilidades sociais, o que, por sua vez, prejudicaria a competência acadêmica.

As crianças que apresentam comportamentos sociais apropriados são hábeis para entender as regras sociais, interagir com pares e adultos e regular suas emoções, especialmente as negativas e, por isso, estariam representadas no status popular (Coie \& Dodge, 1983; Del Prette \& Del Prette, 2005; Miras, 2004). Em contrapartida, as crianças com comportamentos sociais inapropriados (agressivos, disruptivos, timidez etc.) estariam representadas nos status rejeitado e negligenciado por apresentarem um repertório deficitário de habilidades sociais que se refletiria também sobre o desempenho acadêmico (Elliott \& Greham, 1993; Stone \& La Greca, 1990). Para Gresham (200I), a aceitação por pares estaria vinculada a habilidades como: cooperar, oferecer apoio, liderar pares e formar grupos, enquanto que as habilidades de exibir atitudes esnobes, agredir indiretamente, iniciar brigas e romper grupos seriam os principais comportamentos "responsáveis" pela rejeição dos alunos em sala de aula.

Ainda que não se disponha de normas de referência, a validade social das medidas sociométricas, em termos de sua correlação com problemas psicológicos, tem sido demonstrada por diferentes estudos (Coie \& Kupersmidt, 1983; Parker \& Asher, 1987). Na literatura especializada ainda são poucos os estudos que investigam o status sociométrico de crianças em idade escolar, sendo ainda mais escassos aqueles que investigam programas voltados à melhoria da aceitação dos alunos com dificuldades de aprendizagem por seus pares (Elliott \& Greham, 1993; Gresham, Sugai \& Horner, 200 I; Vaughn \& Hogan, 1990; Vaughn, Mclntosh \& Hogan, 1990). Um estudo conduzido por Molina (2003) mostrou os efeitos do Treinamento em Habilidades Sociais sobre o desempenho social e acadêmico de alunos com dificuldades de aprendizagem. O Treinamento em Habilidades Sociais consistiu em atividades lúdicopedagógicas estruturadas previamente e organizadas em ordem crescente de complexidade. $O$ registro do repertório acadêmico dos alunos foi efetuado por meio de uma avaliação formal das habilidades de leitura, nomeação e ditado, e o de habilidades sociais por meio da auto-avaliação e da avaliação pelos colegas e professores. Os resultados mostraram que o grupo que participou da intervenção em habilidades sociais, além de ter apresentado aumentos percentuais em leitura e escrita, mostrou melhorias acentuadas no repertório social, em termos da auto-avaliação e avaliação pelos professores, e alterações positivas no status de $50 \%$ de seus participantes. Esses resultados fortalecem a idéia da importância da promoção de habilidades sociais em alunos com dificuldades de aprendizagem.

Mesmo tendo sua importância reconhecida, o repertório de habilidades sociais dos alunos não tem sido explorado para a maximização da aprendizagem. Isto ocorre porque há uma tendência em se acreditar que as crianças podem adquirir as habilidades sociais como parte do processo de desenvolvimento ou como produto da educação formal. O desenvolvimento interpessoal pode, no entanto, ser promovido por treinamento de habilidades sociais, que representa um intento direto e sistemático de ensino de estratégias e habilidades interpessoais aos indivíduos com o objetivo 
de melhorar a qualidade de suas interações sociais (Curran, 1979; Del Prette \& Del Prette, 1999; 2005). Neste sentido, o Treinamento em Habilidades Sociais se configuraria como uma alternativa interessante para reduzir a baixa aceitação de alunos com dificuldades de aprendizagem, na medida em que o desenvolvimento do repertório social destas crianças repercutiria positivamente na avaliação pelos colegas, afetando seu autoconceito (representações sobre aspectos como capacidades intelectuais) e o próprio ato de aprender (Garcìa \& Musitu, 1993; Miras, 2004).

Com base nestas considerações, pode-se supor que a promoção de habilidades sociais em crianças com dificuldades de aprendizagem rejeitadas, negligenciadas ou controvertidas geraria avaliações mais positivas por seus pares. Neste sentido, a pesquisa ora relatada buscou elaborar e aplicar um programa de treinamento de habilidades sociais de crianças com dificuldades de aprendizagem simultaneamente incluídas nos status rejeitado, negligenciado ou controvertido; avaliar os efeitos da promoção de habilidades sociais sobre o status sociométrico desses alunos.

\section{Método}

\section{Participantes}

Participaram deste trabalho 24 estudantes de ambos os sexos, sendo doze com dificuldades na aprendizagem da leitura e doze sem dificuldades, todos de duas escolas do ensino fundamental de uma cidade do interior de São Paulo (dezoito alunos da escola $A$ e seis da escola $B$ ). A idade dos alunos variou de nove a 12 anos. Dos 15 alunos com dificuldades de aprendizagem inicialmente apontados pelas professoras (oito da escola $B$ e sete da escola $A$ ), foram selecionados os doze que apresentaram ausência de leitura em uma tarefa com quinze palavras e que, concomitantemente, se enquadraram em um dos status negativos (controvertido, negligenciado e/ou rejeitado), segundo avaliação feita pela pesquisadora com o Protocolo de Indicação Sociométrica (Coie, Dodge \& Copottelli, 1982).
Para compor a amostra de alunos sem dificuldades de aprendizagem foi escolhida, por indicação da diretora da escola, a sala com os alunos de melhores rendimentos acadêmicos do local'. A Tabela I mostra a composição dos grupos de alunos DA (com dificuldades de aprendizagem) e NDA (sem dificuldades de aprendizagem) selecionados pela pesquisadora e o status sociométrico revelado por esses participantes.

O Grupo de Intervenção (Gl), composto por seis alunos com dificuldades de aprendizagem (todos da mesma sala-escola B), passou pelo programa de Treinamento em Habilidades Sociais. Os alunos do GCI e GC2 não passaram por intervenção. Dos sete alunos indicados pela professora do $\mathrm{GCl}$ foram selecionados seis (por sorteio) para compor uma amostra equivalente ao Gl. Para compor o GC2 foram selecionados os doze alunos com altos rendimentos apontados pela professora, os quais serviram como grupo de comparação da diferença ente alunos com e sem dificuldades acadêmicas (grupo de referência). $O$ critério de escolha entre Gl e GCl para passar pelo programa de intervenção foi a disponibilidade imediata da diretora da escola do primeiro grupo.

\section{Instrumento}

Para avaliar as crianças antes e depois da intervenção, o Protocolo de Indicação Sociométrica (Coie, Dodge \& Copottelli, 1982) foi utilizado, no qual se pede que cada criança da classe liste três colegas que ela mais gosta e três que ela menos gosta. Os resultados dessa avaliação produzem dois escores imediatos ("gosto mais" e "gosto menos") e dois posteriores: impacto social (soma dos dois imediatos) e preferência social (diferença entre "gosto mais" e "gosto menos"). Esses indicadores permitem identificar as crianças que se encaixam nos status apresentados ou em nenhum deles (nenhuma categoria).

\section{Procedimento}

A aplicação dos instrumentos de avaliação ocorreu nas escolas dos participantes (A e B), ambas situadas em um município do interior de São Paulo. $\bigcirc$ Trei-

\footnotetext{
I Cabe destacar que na sala dos alunos com dificuldades de aprendizagem existiam estudantes sem dificuldades, porém com rendimento médio e, portanto, não foram incluídos na pesquisa.
} 
Tabela I. Descrição geral dos grupos de alunos selecionados pela pesquisadora após a indicação inicial de suas respectivas professoras.

\begin{tabular}{|c|c|c|c|c|}
\hline $\begin{array}{l}\text { Rendimento } \\
\text { acadêmico }\end{array}$ & Grupos & $\begin{array}{c}\text { Alunos } \\
\text { indicados pela } \\
\text { professora }\end{array}$ & $\begin{array}{c}\text { Alunos } \\
\text { selecionados pela } \\
\text { pesquisadora }\end{array}$ & $\begin{array}{l}\text { Status dos alunos } \\
\text { selecionados pela } \\
\text { pesquisadora }\end{array}$ \\
\hline \multirow{10}{*}{$\mathrm{DA}$} & \multirow{5}{*}{$\begin{array}{c}\text { Grupo de } \\
\text { intervenção } \\
\text { (GI) }\end{array}$} & \multirow{5}{*}{8 alunos } & \multirow{5}{*}{6 alunos } & Controvertidos (1) \\
\hline & & & & Negligenciados (1) \\
\hline & & & & Populares (0) \\
\hline & & & & Rejeitados (4) \\
\hline & & & & Nenhuma categoria $(0)$ \\
\hline & \multirow{5}{*}{$\begin{array}{c}\text { Grupo } \\
\text { Controle } 1 \\
(\mathrm{GC} 1)\end{array}$} & \multirow{5}{*}{7 alunos } & \multirow{5}{*}{6 alunos } & Controvertidos $(0)$ \\
\hline & & & & Negligenciados (1) \\
\hline & & & & Populares (0) \\
\hline & & & & Rejeitados (5) \\
\hline & & & & Nenhuma categoria $(0)$ \\
\hline \multirow{5}{*}{ NDA } & \multirow{5}{*}{$\begin{array}{c}\text { Grupo } \\
\text { Controle } 2 \\
\text { (GC2) }\end{array}$} & \multirow{5}{*}{12 alunos } & \multirow{5}{*}{12 alunos } & Controvertidos $(0)$ \\
\hline & & & & Negligenciados $(0)$ \\
\hline & & & & Populares (4) \\
\hline & & & & Rejeitados $(0)$ \\
\hline & & & & Nenhuma categoria (8) \\
\hline
\end{tabular}

namento em Habilidades Sociais (THS) foi conduzido na sala dos participantes no horário de aula.

Fase I: Avaliação pré-intervenção

Após a assinatura do termo de consentimento livre, esclarecido pelos pais ou responsáveis, os alunos com dificuldades de aprendizagem (indicados pelas professoras do $\mathrm{Gl}$ e $\mathrm{GCl}$ ) foram avaliados pela pesquisadora em uma tarefa que envolvia a leitura de 15 palavras simples (bule, gaveta, sino, rádio, suco, goiaba, rio, fogo, caju, tijolo, rede, macaco, mapa, roda e cabide), apresentadas em folhas sulfite com letra arial fonte 16. Os alunos que apresentaram ausência completa de leitura (porcentagem zero) nessa tarefa foram inicialmente selecionados pela pesquisadora.

Em seguida, esses alunos foram avaliados pela pesquisadora por meio do Protocolo de Indicação Sociométrica (Coie, Dodge \& Copottelli, 1982). Para a aplicação deste instrumento, realizada individualmente, perguntava-se à criança: a) Quais as três crianças de sua sala que você gosta mais, que você está sempre junto, que gosta de brincar, conversar? e - Quais as três crianças de sua sala que você não gosta, não fica junto, não brinca, não conversa? Os nomes citados e a avaliação correspondente eram circulados no protocolo. Assim que o participante terminava a avaliação, enfatizava-se a possibilidade de mudança nas relações de amizade como forma de minimizar efeitos indesejáveis da própria avaliação. Lembrando que os alunos com dificuldades de aprendizagem que se enquadravam nos status negligenciado, controvertido ou rejeitado foram selecionados para compor o $\mathrm{Gl}$ e o $\mathrm{GCl}$.

Fase II: Intervenção em Habilidades Sociais

O Treinamento em Habilidades Sociais abrangeu 10 sessões de uma hora e meia cada e foi realizado na sala de aula do Grupo de Intervenção (GI). Foram utilizados procedimentos lúdico-pedagógicos associados a técnicas de modelação, instrução, solução de problemas, feedback, role-playing etc. (Caballo, 1987; Del Prette \& Del Prette, 200I; Gresham, 1995; 200I). O programa de promoção 
de habilidades sociais foi previamente planejado tendo em vista uma organização das habilidades consideradas significativas para os alunos em idade escolar (Caldarella \& Merrell, 1997; Givvin, Stipek, Salmon \& Mac Gyvers, 1998; Gresham, 200I; Gresham \& Elliott, 1990). As sessões, previamente construídas pela pesquisadora, incluíam diferentes vivências, baseadas em Del Prette e Del Prette (1999), incluindo três partes: parte inicial - verificação de tarefas de casa e atividades de aquecimento que já podiam incluir algumas habilidades básicas (observação, atenção etc.); parte central - aplicação de procedimentos relacionados aos objetivos do programa, por exemplo, treinamento das habilidades de elogiar, perguntar, discordar etc.; parte final - avaliação da sessão e atribuição de tarefas de casa. Estas tarefas consistiam em atividades atribuídas aos participantes para promover a generalização das aquisições. Por exemplo, se em uma sessão era ensinado o comportamento de elogiar, era proposta como tarefa que os participantes emitissem três elogios para pessoas próximas (pai, mãe, colegas de sala etc) naquela semana. No início da sessão seguinte, o participante relatava a experiência.

Fase III: Avaliação pós-intervenção

Os alunos foram novamente avaliados pela pesquisadora por meio do Protocolo de Indicação Sociométrica (Coie, Dodge \& Copottelli, 1982).

\section{Resultados e Discussão}

Os dados envolvendo os três colegas indicados (em termos de "mais gosto" e "menos gosto") foram tratados para identificação dos alunos rejeitados, controvertidos, populares e negligenciado da sala de aula e para verificação da situação dos participantes com e sem dificuldades de aprendizagem deste trabalho, antes e depois da intervenção. Este tratamento envolveu a computação dos escores de "gosto muito" (GM), "não gosto" (NG), "preferência social" (PS) e "impacto social" (IS). Os escores GM e NG foram baseados no número total de indicações que cada aluno recebeu, enquanto os escores de PS e IS foram obtidos através do seguinte cálculo: $\mathrm{PS}=\mathrm{GM}-\mathrm{NG}$ $\mathrm{e} I \mathrm{I}=\mathrm{GM}+\mathrm{NG}$, respectivamente. A normalização dos dados da sala de aula para cada um destes indicadores foi feito por meio da fórmula: $[(x-\bar{x}) / \sigma]$, onde $x=$ valor obtido pelo participante em termos de GM, NG, PS e IS; $\bar{x}=$ valor médio de todos os participantes da sala em termos de GM, NG, PS e IS; $\sigma=$ desvio padrão dos alunos da sala. Assim, foi possível a classificação dos alunos, segundo os critérios, em: populares (PS > I; GM > 0 e NG < 0); rejeitados ( $\mathrm{PS}<\mathrm{I}$; $\mathrm{NG}>0$ e $\mathrm{GM}<0$ ); negligenciados (IS < I e GM = 0) e controvertidos (IS > I e GM e NG > 0). Os alunos que não se enquadraram em uma das classificações explicitadas acima permaneceram sem status (nenhuma categoria).

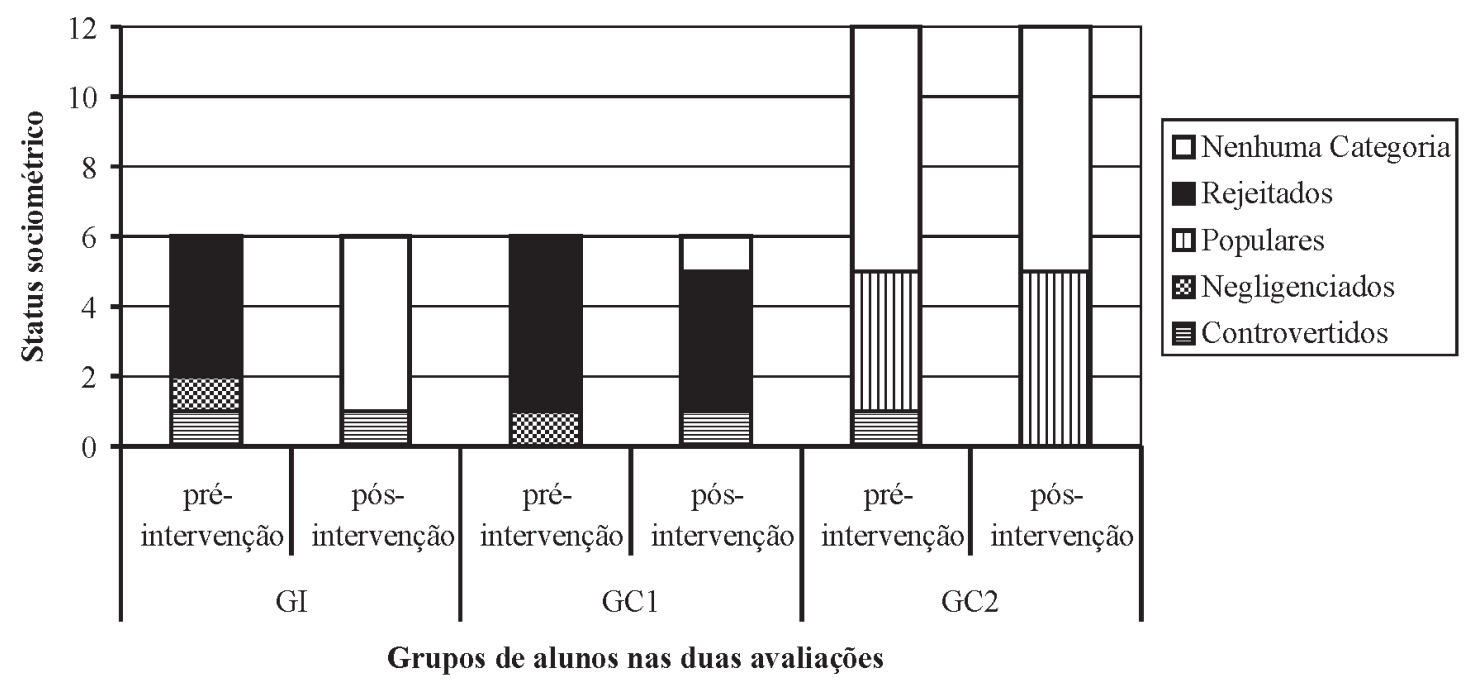

Figura I. Resultados da avaliação inicial (pré-intervenção) e final (pós-intervenção) do Protocolo de Indicação Sociométrica dos grupos 
A Figura I mostra os resultados da avaliação inicial (pré-intervenção) e final (pós-intervenção) do Protocolo de Indicação Sociométrica das crianças com dificuldades de aprendizagem que passaram por intervenção (GI) e daquelas com dificuldades (GCI) e sem dificuldades (GC2) que não passaram por intervenção.

Os dados apresentados na Figura I mostraram que, na pré-intervenção, das crianças com dificuldades de aprendizagem ( $\mathrm{Gl}$ e $\mathrm{GCl}$ ), nove eram rejeitadas (75\%), uma delas era controvertida (8,33\%) e duas eram negligenciadas (16,67\%). Os resultados das crianças sem dificuldades de aprendizagem (GC2) nessa mesma avaliação mostraram que quatro desses alunos eram populares $(33,33 \%)$, um era controvertido $(8,33 \%)$ e os demais não foram incluídos em categorias distintas (58,33\%). Os dados gerais obtidos confirmaram os estudos de Bryan e Sherman (1980), Elliott e Gresham (1993), Stone e La Greca (1990), Vaughn e Hogan (1990) e Vaughn, McIntosh e Spencer-Rowe (1991) que têm mostrado que os alunos com dificuldades de aprendizagem são menos aceitos que seus colegas com desempenho acadêmico satisfatório.

A avaliação por grupo mostrou que, na pré-intervenção, das seis crianças componentes do $\mathrm{Gl}$, quatro eram rejeitadas $(66,66 \%)$, uma era controvertida (13,33\%) e uma era negligenciada (I3,33\%). Na pós-intervenção, apesar de um participante ter continuado a ser repre- sentado no status controvertido (13,33\%), os demais alunos do $\mathrm{Gl}$ deixaram de ser rejeitados e negligenciados (86,66\%), confirmando pesquisas que demonstraram efeitos positivos da promoção de habilidades sociais sobre a aceitação de alunos com dificuldades de aprendizagem por seus colegas de sala de aula (Molina, 2003; Vaughn \& Hogan, 1990; Vaughn, Mclntosh \& Hogan, 1990; Vaughn, McIntosh \& Spencer-Rowe, 199I).

Os dados do $\mathrm{GCl}$ mostram que na pré-intervenção, cinco crianças eram rejeitadas $(83,33 \%)$ e uma era negligenciada (16,67\%); na pós-intervenção, apesar de um participante ter deixado de ser negligenciado $(16,67 \%)$ e um não ter sido incluído um uma categoria distinta (16,67\%), quatro participantes do $\mathrm{CCl}$ $(66,67 \%)$ continuaram sendo representados no status rejeitado. Quanto aos doze participantes sem dificuldades de aprendizagem do GC2 nas duas avaliações, uma elevada porcentagem dos alunos desse grupo foi enquadrada no status popular $(33,33 \%$ antes e $41,66 \%$ após a intervenção) e em nenhuma categoria (58,33\% antes e após a intervenção). Os dados do GCI e GC2 confirmam os achados de Coie e Dodge (1983) sobre o grau de estabilidade de um sujeito dentro de um status.

A Tabela 2 mostra o desempenho geral dos grupos na comparação entre pré e pós-intervenção, indicando mudanças positivas (melhora de status), negativas (piora de status) e sem mudanças (manutenção do status).

Tabela 2. Porcentagem de alunos dos três grupos que permaneceram no status original e dos que obtiveram mudanças positivas e negativas na pós em comparação com a pré-intervenção

\begin{tabular}{ccccc}
\hline Grupos & $\mathrm{N}$ & $\begin{array}{c}\text { Mudanças } \\
\text { positivas }\end{array}$ & $\begin{array}{c}\text { Mudanças } \\
\text { negativas }\end{array}$ & Sem mudanças \\
\hline GI & 06 & $83,33 \%$ & ----- & $16,66 \%$ \\
(DA com intervenção) & & & & $50 \%$ \\
\hline GC1 & 06 & $33,33 \%$ & $16,67 \%$ & \\
\hline
\end{tabular}

\section{$\mathrm{GC} 2$}

(NDA sem intervenção)

$12 \quad 16,67 \% \quad-----\quad 83,33 \%$

Legenda: $\mathrm{DA}=$ alunos com dificuldade de aprendizagem; $\mathrm{NDA}=$ alunos sem dificuldade de aprendizagem; $\mathrm{N}=$ número de participantes. 
Os dados mostraram que a maioria dos alunos do GI apresentou mudanças positivas em seus status $(83,33 \%)$, enquanto que a maior parte dos estudantes do $\mathrm{GCl}$ e GC2 permaneceu com o mesmo status da pré-intervenção (50\% e $83,33 \%$, respectivamente). A estabilidade revelada pelo $\mathrm{GCl}$, aliada à piora de status de um participante desse grupo, e a melhora no status da maioria dos alunos do Gl, sugeriram que a melhor aceitação pelos colegas pode ter sido um reflexo de relações interpessoais mais satisfatórias entre estes e os estudantes avaliados, indicando benefícios do Treinamento em Habilidades Sociais sobre a avaliação do repertório social de alunos com dificuldades de aprendizagem.

Conforme Givvin, Stipek, Salmon e Mac Gyvers (1998), comportamentos sociais adequados geram avaliações mais positivas pelos pares na escola. Isto, porque em todos os níveis de escolaridade os alunos atribuem uma importância primordial aos aspectos afetivos e de relação interpessoal em sala de aula.

Mesmo considerando o número reduzido de participantes da pesquisa ora relatada, o programa de intervenção conduzido com o Gl se mostrou efetivo e coerente com as metas previamente estabelecidas, apontando o Treinamento em Habilidades Sociais como uma alternativa para reduzir a baixa aceitação de alunos com dificuldades de aprendizagem no ensino brasileiro. Uma hipótese explicativa para a positividade dos resultados com o $\mathrm{Gl}$ aponta que o desenvolvimento do repertório social das crianças desse grupo repercutiu positivamente na avaliação pelos colegas de sala de aula, diminuindo a porcentagem de rejeição encontrada na avaliação inicial daqueles estudantes. Uma outra hipótese diz respeito ao fato da inclusão de pares com bons rendimentos acadêmicos e sociais na intervenção aumentar a probabilidade de seguimentos de modelos pelos alunos com dificuldades de aprendizagem, auxiliando a generalização das mudanças vivenciadas no treinamento (Vaughn, 1991).

Uma outra explicação para a alteração de status registrada pelos estudantes do $\mathrm{Gl}$ nas duas avaliações refere-se ao fato de todos os alunos desta sala terem participado do programa de intervenção, o que pode ter contribuído para que os pares "socialmente normais" avaliassem de maneira mais positivas seus cole- gas com dificuldades de aprendizagem. Esse fato pode ainda ter sido reforçado pelo comando verbal emitido pela pesquisadora no momento da aplicação do instrumento utilizado, "Assim que o participante terminava a avaliação, enfatizava-se a possibilidade de mudança nas relações de amizade como forma de minimizar efeitos indesejáveis da própria avaliação".

Novas pesquisas visando avaliar o status sociométrico de crianças com dificuldades de aprendizagem com um maior número de participantes, possibilitando a inclusão de avaliações estatísticas, são necessárias e relevantes à área. A inclusão de um número razoável de participantes na pesquisa pode promover um rol maior de conhecimento sobre os tipos de déficits sociais encontrados em alunos com dificuldades de aprendizagem, auxiliando a descoberta de procedimentos de intervenção mais efetivos para estes estudantes.

A realização das pesquisas nesta área exigirá iniciativas para a resolução dos diversos empecilhos típicos em trabalhos conduzidos com seres humanos. Os problemas mais comuns referem-se à falta de assiduidade dos alunos na escola e à dificuldade da obtenção da assinatura do termo de consentimento pelos pais. Assim, torna-se emergencial a necessidade de programas voltados a uma maior participação dos pais sobre as decisões relacionadas à educação de seus filhos, conscientizando-se acerca de suas dificuldades acadêmicas e da necessidade de recursos específicos para o ideal desenvolvimento das potencialidades destes alunos.

Um outro ponto a ser considerado é que, apesar de representarem uma variável importante para o ensino e para a aprendizagem, as habilidades sociais têm constituído um subproduto esperado mais do que um objetivo planejado para a educação escolar (Asher \& Rose, 1999). Isto ocorre porque, de certa forma, há uma tendência em se acreditar que tais habilidades são adquiridas espontaneamente no processo de desenvolvimento. Por outro lado, a promoção de habilidades sociais em sala, que envolveria um certo domínio conceitual e metodológico dessa área por parte do professores, passa a ser um produto incidental de sua experiência cotidiana e, quando ocorre, não se respalda em concepções e estratégias ade- 
quadas de ensino (Del Prette \& Del Prette, 1997). Assim, a concretização dos objetivos de promoção de interações sociais educativas, em sala de aula, requer a elaboração de novas pesquisas voltadas para a construção e aplicação de programas que orientem o professor no sentido de (Del Prette, Del Prette, Pontes \& Torres, 1998): compreender o papel das habilidades sociais para o desempenho acadêmico e social dos alunos, identificar a relação entre seu comportamento e o dos alunos, ampliar seu repertório de habilidades sociais e utilizar procedimentos para promoção de desenvolvimento interpessoal em sala.

Programas voltados à promoção de habilidades sociais em sala poderiam facilitar a aprendizagem acadêmica e social de estudantes com dificuldades de aprendizagem, amenizando os efeitos do fracasso escolar.

\section{Referências}

Almeida, S. F. C., Rabelo, L. M., Cabral, V. S., Moura, E. R. O., Barreto, M. S. F., \& Barbosa, H. (1995). Concepções e práticas de psicólogos escolares acerca das dificuldades de aprendizagem. Psicologia: Teoria e Pesquisa, II, | I 7- I 34.

Asher, S. R., \& Coie, S. D. (Eds.). (1990). Peer rejection in childhood. New York: Cambridge University Press.

Asher, S. R., \& Rose, A. J. (1999). Como promover a adaptação social e emocional das crianças com os colegas. Em P. Salovey \& D. J. Sluyter (Orgs.), Inteligência emocional da criança: Aplicaçóes na educação e no dia-a-dia (pp. 247-289). Rio de Janeiro: Campus.

Bryan, T., \& Sherman, R. (1980). Immediate impressions of nonverbal ingratiation attempts by learning disabled boys. Learning Disabilities, 3, 19-28.

Caballo, V. E. (1987). Teoría, evaluación y entrenamiento de las habilidades sociales. Valencia: Promolibro.

Caldarella, P., \& Merrell, K. W. (1997). Common dimensions of social skills of children and adolescents: A taxonomy of positive behaviors. School Psychology Review, 26, 264-278.

Coie, J. D., \& Dodge, K. A., (1983). Continuities and changes in children's social status: A five year longitudinal study. MerrilPalmer Quarterly, 29, 26I-28I.
Coie, J. D., Dodge, K. A., \& Coppotelli, H. (I982). Dimensions and types of social status: A cross-age perspective. Developmental Psychology, 18, 557-570.

Coie, J. D., \& Kupersmidt, J. B. (1983). A behavioral analysis of emerging social status in boy's group. Child Development, 54, $1400-1416$.

Curran, J. (1979). Pandora's box reopened? The assessment of social skills. Journal of Behavior Assessment, 2, 55-7I.

Davis, C., Silva, M. A., \& Espósito, Y. (1989). Papel e valor das interações sociais em sala de aula. Cadernos de Pesquisa, 7I, 49-54.

Del Prette, A., \& Del Prette, Z. A. P. (1997). Habilidades sociais e construção de conhecimento em contexto escolar. Em D. R. Zamignani (Org.), Sobre Comportamento e Cognição: A Aplicação da Análise do Comportamento e da Terapia Cognitivocomportamental no Hospital Geral e nos Transtornos Psiquiátricos (pp. 234-250). Santo André: ARBytes.

Del Prette, A., \& Del Prette, Z. A. P. (200I). Psicologia das relações interpessoais: Vivências para o trabalho em grupo. Petrópolis: Vozes.

Del Prette, A., Del Prette, Z. A. P., Pontes, A. C., \& Torres, A. C. (1998). Efeitos de um programa de intervenção sobre aspectos topográficos das habilidades sociais de professores. Psicologia Escolar e Educacional, 2, II-22.

Del Prette, Z. A. P., \& Del Prette, A. (1999). Psicologia das Habilidades Sociais: Terapia e Educação. Rio de Janeiro: Vozes.

Del Prette, Z. A. P., \& Del Prette, A. (2003). Habilidades sociais e dificuldades e aprendizagem: Teoria e pesquisa sob um enfoque multimodal. Em A. Del Prette \& Z. A. P. Del Prette (Orgs.), Habilidades sociais desenvolvimento e aprendizagem: Questões conceituais, avaliação e intervenção (pp. 167-206). Campinas: Alínea.

Del Prette, Z. A. P., \& Del Prette, A. (2005). Treinamento de Habilidades Sociais na Infância: Teoria e Prática. Rio de Janeiro: Vozes.

Elliott, S. N., \& Greham, F. M. (1993). Social skills intervention for children. Behavior Modification, 17, 287-313.

Fad, K. S. (1989). The fast track to success: Social behavioral skills. Intervention in School and Clinic, 3, 39-42.

Frentz, F., Gresham, F. M., \& Elliott, S. N. (1990). Popular, controversial, neglected, and rejected adolescents: Contrasts of social competence and achievement differences. Journal of School Psychology, 23, 109-120. 
Garcia, F. J., \& Musitu, G. (1993). Rendimiento acadêmico y autoestima em el Ciclo Superior de EGB. Revista de Psicologia da la Educacíon, 4, 73-87.

Givvin, K. B., Stipek, D. J., Salmon, J. M., \& Mac Gyvers, V. L. (1998). In the eyes of the beholder: students' and teacher's judgments of students' motivation (texto mímeo).

Gresham, F. M. (1992). Social skills and learning disabilities: Causal, concomitant or correlational? School Psychological Review, 2I, 348-360.

Gresham, F. M. (1995). Best practices in social skills training. Em A. Thomas \& J. Grimes (Orgs.), Best Practices in School Psychology-III (pp. I02 I- 1030). Washington DC: The National Association of School Psychologists.

Gresham, F. M. (200I). Assessment of social skills in children and adolescents. Em W. W. Andrews \& H. Saklofske (Orgs.), Handbook of Psychoeducational Assessment: Ability, Achievement and Behavior in Children (pp. 325-355). San Diego: Academic Press.

Gresham, F. M., \& Elliott, S. N. (1989). Social skills as a primary learning disability. Journal of Learning Disabilities, 22, I20-I24.

Gresham, F. M., \& Elliott, S. N. (1990). Social skills rating system. Circle Pines: American Guidance Service.

Gresham, F. M., Sugai, G., \& Horner, R. H. (200I). Interpreting outcomes of social skills training for students with highincidence disabilities. Exceptional Children, 67, 331-344.

Malecki, C. K., \& Elliott, S. N. (2002). Children's social behaviors as predictors of academic achievement: A longitudinal analysis. School Psychology Quarterly, I7, I-23.

Maluf, M. R., \& Bardelli, C. (199I). As causas do fracasso escolar na perspectiva de professoras e alunos de uma escola de primeiro grau. Psicologia: Teoria e Pesquisa, 7, 255-262.

Marturano, E. M., Linhares, M. B. M., \& Parreira, V. L. C. (1993). Problemas emocionais associados a dificuldades na aprendizagem escolar. Medicina Ribeirão Preto, 26, I6I-I75.

Marturano, E. M., \& Loureiro, S. M. (2003). O desenvolvimento sócio-emocional e as queixas escolares. Em A. Del Prette \& Z. A. P. Del Prette (Orgs.), Habilidades sociais, desenvolvimento e aprendizagem: Questões conceituais, avaliação e intervenção (pp. 259-29I). Campinas: Alínea

McClelland, M. M., Morrison, F. J., \& Holmes, D. L. (2000). Children at risk for early academic problems: The role of learning-related social skills. Early Childhood Research Quarterly, 15, 307-329.
Melo, M. H. S. (2004). Crianças com dificuldades de interação no ambiente escolar: Uma intervenção multifocal. Tese de Doutorado, Programa de Pós-Graduação em Psicologia Clínica, Universidade de São Paulo, São Paulo.

Merrell, K. W. (1993). School social behavior scales. Austin: Pro-ED.

Miras, M. (2004). Afetos, emoções, atribuiçõos e expectativas: O sentido da aprendizagem escolar. Em C. Coll, J. Palácios \& A. Marchesi (Orgs.), Desenvolvimento psicológico e educação: Psicologia da Educação Escolar 2 (pp. 209-222). Porto Alegre: Artmed.

Molina, R. C. M. (2003). Habilidades sociais e dificuldades de aprendizagem: Uma análise funcional. Dissertação de Mestrado, Programa de Pós-Graduação em Educação Especial, Universidade Federal de São Carlos, São Carlos.

Molina, R. C., \& Del Prette, Z. A. P. (2002). Habilidades sociais e dificuldades de aprendizagem: Uma análise funcional. Anais do V Congresso de Pesquisa e Educação da Região Sudeste: Tendências e Desafios (CDD 370), ISBN 85-903167-I-8, Águas de Lindóia, SP.

Newcomb, A., Bukowski, W., \& Patee, L. (1993). Children's peer relations: A meta-analytic review of popular, rejected, neglected, controversial, and average sociométrico status. Psychological Bulletin, I/3, 306-347.

O'Shaughnessy, T. E., Lane, K. L., Gresham, F. M., \& BeebeFrankenberger, M. E. (2002). Students with at risk for learning and emotional-behavioral difficulties: An integrated system of prevention and intervention. Em K. L. Lane, F. M. Gresham \& T. E. O'Shaughnessy (Orgs.), Children with or at risk for emotional and behavioral disorders (pp. 3-17). Boston: Allyn \& Bacon.

Parker, J. G., \& Asher, S. R. (1987). Peer relation and later personal adjustement: Are low accepted children at risk? Psychological Bulletin, 102, 357-389.

Pontecorvo, C. (sd). Social context, semiotic mediation and forms of discourse in constructing knowledge at school (Mimeo).

Romero, A. (1995). As relações sociais das crianças com dificuldades de aprendizagem. Em C. Coll, J. Palácios \& A. Marchesi (Orgs.), Desenvolvimento psicológico e educação: Necessidades educativas especiais e aprendizagem escolar (pp. 7I-82). Porto Alegre: Artes Médicas.

Saint-Onge, M. (1999). O ensino na escola: O que é, como se faz. São Paulo: Loyola.

Sánchez, J. N. G. (2004). Dificuldades de Aprendizagem e Intervenção Psicopedagógica. Porto Alegre: Artmed. 
Sisto, F. F., Boruchovitch, E., Fini, L. D. T., Brenelli, R. P., \& Martinelli, S. C. (2004). Dificuldades de aprendizagem no contexto psicopedagógico. São Paulo: Vozes.

Stone, W. L., \& La Greca, A. M. (1990). The social status of children with LD: A reexamination. Journal of Learning Disabilities, 23, 32-37.

Swanson, H. L., \& Malone, S. (1992). Social skills and learning disabilities: A meta-analysis of the literature. School Psychology Review, 21, 427-443.

Vaughn, S. (199I). Social skills enhancement in students with learning disabilities. Learning about Learning disabilities. Copyright by Academic Press.

Vaughn, S., \& Hogan, A. (1990). Social competence and learning disabilities: A prospective study. Em H. L. Swanson e B. K.
Keogh, (Orgs.), Learning Disabilities: Theoretical and Research Issues (pp. I75-191). New Jersey: Lawrence Erlbaum.

Vaughn, S., Mclntosh, R., \& Hogan, A. (1990). Why social skills training doesn 't work: An alternative model. Em T. Scruggs e B. Y. L. Wong (Eds.), Intervention in Learning Disabilities. Springer Verlag Publ.

Vaughn, S., Mclntosh, R., \& Spencer-Rowe, J. (I99I). Peer rejection is a stubborn thing: Increasing peer acceptance of rejected students with learning disabilities. Learning Disabilities Research \& Practice, 6, 83-88.

Vigotsky, L. S. (2003). Psicologia Pedagógica (edição comentada). Porto Alegre: Artmed.

Weiss, M. L. L. (1992). Psicopedagogia clínica - uma visão diagnóstica. Porto Alegre: Artes Médicas.

Recebido em: 29/0I/2007

Revisado em: 13/06/2007

Aprovado em: 14/09/2007

Sobre os autores:

Renata Cristina Moreno Molina (rcmmpsi@yahoo.com.br) - Doutora em Educação Especial pelo Programa de Pós-Graduação em Educação Especial da Universidade Federal de São Carlos - UFSCar - SP

Almir Del Prette (adprette@power.ufscar.br) - Professor Titular do Departamento de Psicologia da Universidade Federal de São Carlos - UFSCar - SP e orientador do Programa de Pós-Graduação em Educação Especial.

Endereço para contato:

Renata Cristina Moreno Molina

Rua Carlos Gomes, 3.67I,

Bairro Santa Angelina - CEP 14.802-165 Araraquara-SP 